\title{
Poststroke Epilepsy: A Hospital-based Study
}

\author{
Agnibha Maiti ${ }^{1}$, Soumitro Ghosh ${ }^{2}$, Anup K Bhattacharya ${ }^{3}$
}

\begin{abstract}
Introduction: Poststroke epilepsy (PSE) is a very important complication after cerebrovascular accident (CVA). Stroke is responsible for $10 \%$ of all seizures and about $55 \%$ of newly diagnosed epilepsy in elderly population. It may lead to longer hospitalization, worse immediate functional outcome, and high mortality.

Aims and objectives: We have studied 369 CVA patients from July 2017 to June 2019 to study the prevalence of epilepsy in stroke survivors, risk factors, and clinical manifestation of PSE.

Materials and methods: For this purpose, detailed clinical examination, CT scan brain, MRI brain, blood glucose estimation, serum lipid profile, and EEG were carried out.

Observation: Among 369 CVA patients, 34 presented with seizure: 22 patients with early seizure (within 2 weeks) and 12 with late seizure (after 2 weeks). Most of the patients were greater than 60 years of age (27 patients). The male:female ratio was 22:12. Among 34 patients, 26 patients were of cerebral hemorrhage and 8 patients were of cerebral infarct. History of hypertension was present in 29 patients. Majority of PSE patients were diabetic (24 out of total 34). Cortical involvement was present in 29 patients, and in 5 patients there was subcortical involvement. Among 34 patients, 26 experienced focal onset seizure; 5 patients had generalized seizure; and 3 experienced status epilepticus.

Conclusion: Physician should always treat stroke survivors with the risk of PSE in mind. While considerable difficulties exist in designing and conducting clinical studies of PSE, such as high heterogeneity of stroke survivors, further studies are necessary to explore primary and secondary prevention of PSE.
\end{abstract}

Keywords: Antiepileptic drug, Blood-brain barrier, Early seizure, Late seizure, Poststroke epilepsy, Poststroke seizure.

Bengal Physician Journal (2019): 10.5005/jp-journals-10070-6121

\section{INTRODUCTION}

Poststroke epilepsy (PSE) is a significant and well-established clinical issue in patients who survive stroke. In a total of 34 longitudinal studies which was done over 1 lakh patients, 7\% developed PSE. ${ }^{1}$ Poststroke epilepsy has been shown to account for 30 to $49 \%$ of all new onset seizure (Assis et al., 2015; Stefan et al., 2014). The occurrence of PSE often leads to fatal outcome in patients who survived after stroke ${ }^{2-4}$ (Bladin et al.; Labovitz et al.; Arboix et al.). Poststroke epilepsy often recurs in a large percentage of patients. In a study done over 360 days, Tanaka et al., 2015 found that PSE recurred in $30 \%$ patients. Depending on the result of a number of epidemiological studies, it can be said incidence rate of epilepsy is more in hemorrhagic stroke than ischemic stroke, with about $10-20 \%$ of patients developing PSE after hemorrhagic stroke compared with $2-14 \%$ after ischemic stroke. ${ }^{5-7}$ Seizure may occur at a close proximity after stroke (acute symptomatic, provoked, or early seizure) or after a few days interval following stroke (late seizure). According to the International League Against Epilepsy, early seizure is defined as seizure occurring within 7 days of cerebrovascular accident (CVA) and late seizure is defined as seizure occurring after 7 days of CVA. ${ }^{8,9}$ After an incidence of stroke, neuronal injury may occur from different causes, such as dysfunction in metabolism, decreased perfusion in brain, glutamate-induced excitotoxicity, malfunction of different ion channels, activation of astrocyte, inflammation in neurons leading to breakage of blood-brain barrier in the acute phase (Myint et al.; Reddy et al.) ultimately which leads to early seizure. ${ }^{10}$ However, the mechanism behind late seizure is different and may be secondary to gliotic scarring, chronic inflammation, angiogenesis, neurodegeneration, neurogenesis, axonal and synaptic sprouting, selective neuronal loss, and altered synaptic plasticity. It has been

\author{
1,2 Department of Medicine, Institute of Postgraduate Medical \\ Education and Research and Seth Sukhlal Karnani Memorial Hospital, \\ Kolkata, West Bengal, India \\ ${ }^{3}$ Department of Medicine, Agartala Govt Medical College, Tripura, \\ India
}

Corresponding Author: Agnibha Maiti, Department of Medicine, Institute of Postgraduate Medical Education and Research and Seth Sukhlal Karnani Memorial Hospital, Kolkata, West Bengal, India, Phone: +91 9477075971, e-mail: agnivam@gmail.com

How to cite this article: Maiti A, Ghosh S, Bhattacharya AK. Poststroke Epilepsy: A Hospital-based Study. Bengal Physician Journal 2019;6(3):51-54.

\section{Source of support: Nil}

Conflict of interest: None

observed that PSE stands for about $11 \%$ of all adult epilepsy cases above the age of 60 years. However, there is lack of data which can be relied on regarding most important aspect of PSE. Recently, many researchers have come forward with much interest to do study on PSE. Hemorrhagic stroke, severity of initial neurodeficit, patients of older age group, involvement of cerebral cortex, positive family history of seizure, certain genetic factors carry a higher risk of PSE. However, stroke severity judged clinically irrespective of stroke scale used is a major determinant in the development of PSE. $6,7,11-13$ Electroencephalography can be used as an important diagnostic tool which can detect interictal or ictal abnormalities, especially in nonconvulsive seizures and nonconvulsive status epilepticus. Data collected from various sources clearly indicate that all antiepileptic drugs (AEDs) have more or less similar efficacy in treating patient of PSE. Levetiracetam and lamotrigine are the most commonly used AED in PSE. 


\section{Aims and Objectives}

To study the prevalence of epilepsy in stroke survivors and also to find out potential risk factors of PSE.

\section{Inclusion criteria}

Patients suffering from CVA.

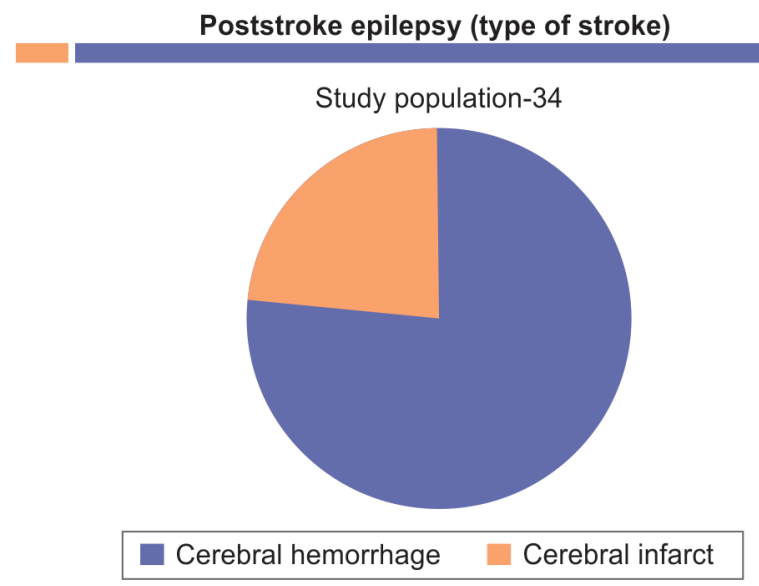

Fig. 1: Cerebral hemorrhage-26; Cerebral infarct-8

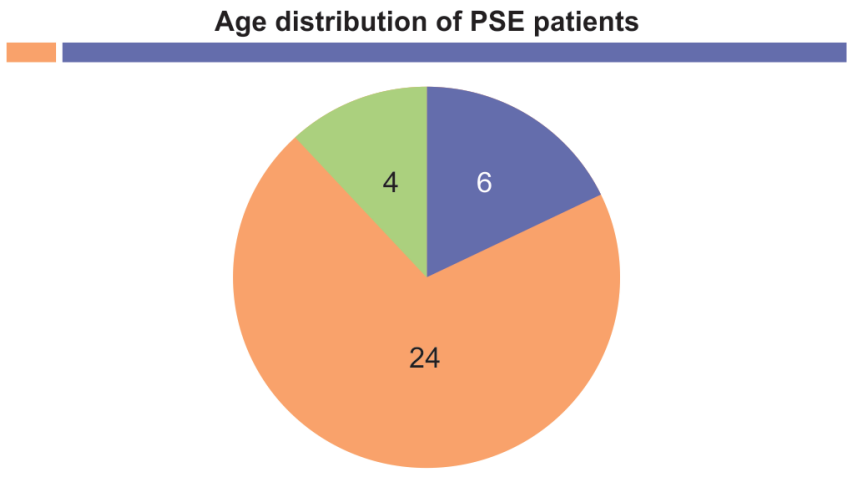

Age between 30 and $60 \square$ Age between 60 and 80 Age greater than 80

Fig. 3: Age between 30 and $60=6$ patients; age between 60 and $80=$ 24 patients; age greater than $80=4$ patients

\section{Exclusion criteria}

- Known epileptic patients already on medication.

- Previous history of CVA.

- Chronic systemic diseases.

- Any known malignancy.

- Any known psychiatric illness.

\section{Prevalence of early and late seizure}

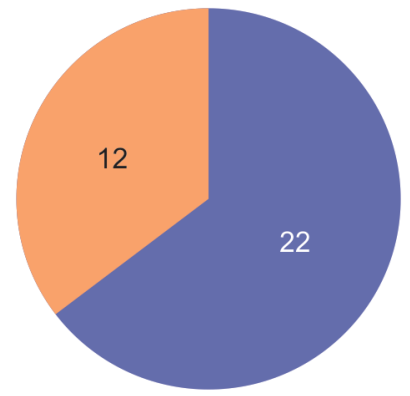

Early-onset seizure (within 1 week) Late-onset seizure (after 1 week)

Fig. 2: 22 presented with early-onset seizure and 12 presented with late-onset seizure

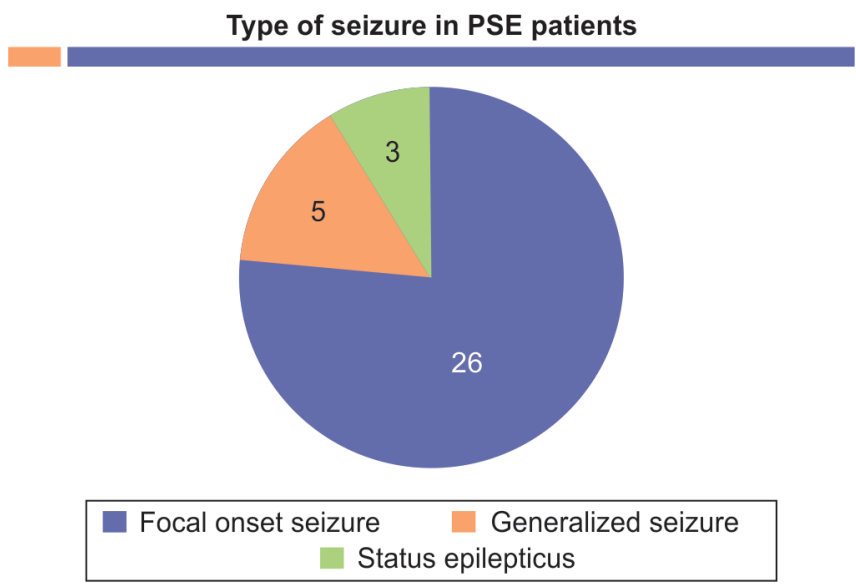

Fig. 4: Majority presented with focal onset seizure, 5 presented with generalized seizure, 3 presented with status epilepticus

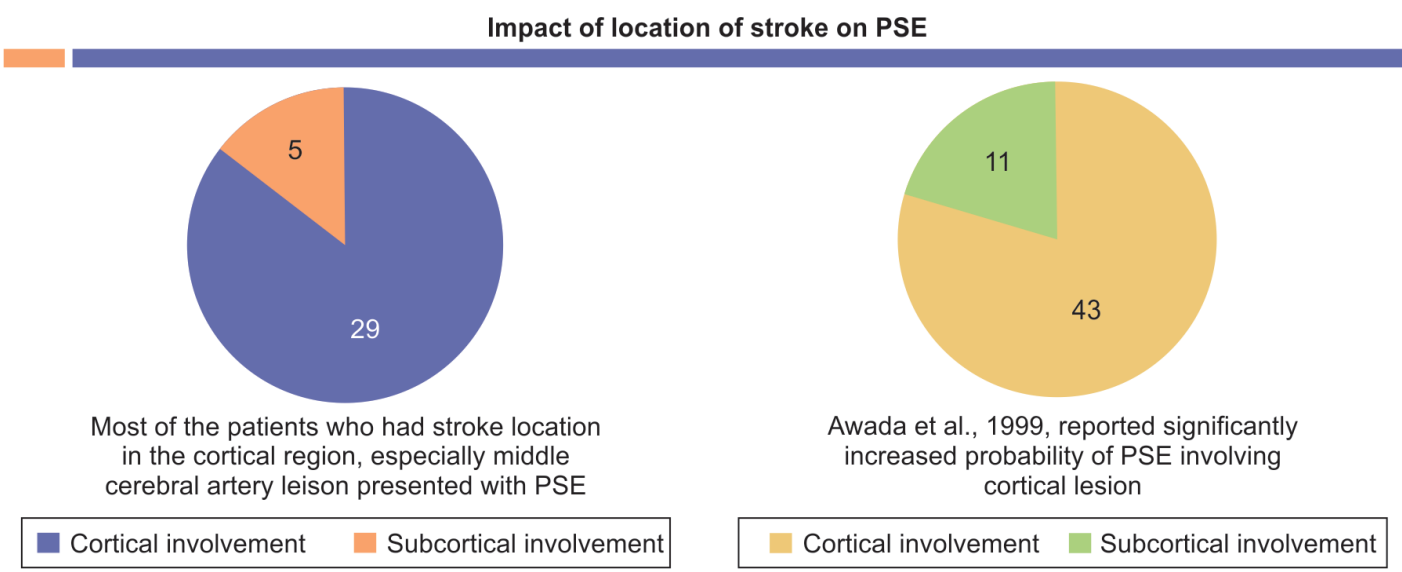

Fig. 5: Cortical involvement $=29$ patients; subcortical involvement $=5$ patients 


\section{Relevant Examination and Investigations}

- Detailed clinical examination.

- Computed tomography scan brain.

- Magnetic resonance imaging brain.

- Blood glucose estimation;

- Serum lipid profile;

- Electroencephalography.

We evaluated (Figs 1 to 7 )

- Type of patients-Those suffering from CVA.

- Site of study_Department of Medicine, Institute of Postgraduate Medical Education and Research;

- Total number of CVA patients-369;

- Number of patients suffered PSE-34;

- Sex: male:female-22:12;

- Age:

- Youngest age of presentation-43 years and 4 months.

- Mean age of presentation-60.33 \pm 6.54 years.

\section{Discussion}

We have found different levels of risk factors for PSE. Most of the patients who had stroke location in the cortical region especially middle cerebral artery lesion presented with PSE. Awada et al., 1999 and Silverman et al., 2002 reported significantly increased

\section{EEG Abnormalities}

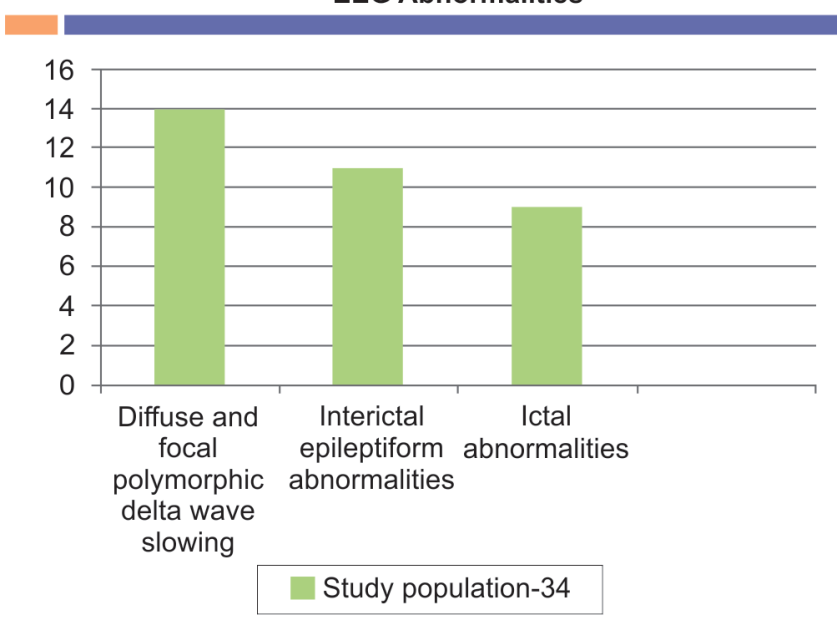

Fig. 6: Majority (79.6\%) of PSE patients had cortical involvement probability of PSE involving cortical lesion. Data collected from various studies clearly indicate that there is more incidence of PSE in hemorrhagic stroke patients rather than ischemic stroke ${ }^{6,7}$ (Arntz et al.; Burn et al.). In our study, we have also found $76.4 \%$ PSE patients were hemorrhagic in nature. The mechanism behind PSE following hemorrhage is not very clear. One probable mechanism may be deposition of hemosiderin which ultimately leads to cerebral irritation causing seizure (Silverman et al., 2002). There is very high probability of PSE in subarachnoid hemorrhage as extent of damage of cerebral cortex is often very severe. Poststroke epilepsy often leads to severe initial neurologic damage after stroke. In an important study done by Conrad et al., interesting finding was that there is much more likelihood of PSE in patients who have high National Institute of Health Stroke Scale. In the patient age group greater than 60 years, we found incidence of PSE was very high. In contrast to our study, Graham et al., 2013; Tanaka et al., 2015; Werhahn et al., 2011 found that younger age group carries more risk of developing PSE. Similar to our study, The National General Practice Study of Epilepsy cerebrovascular disease leads to approximately $15 \%$ of newly diagnosed epilepsy, and among them $50 \%$ was elderly. ${ }^{14}$ Majority of our patients who presented with PSE was diabetic and hypertensive. Ohman, 1990; Wang et al., 2013 made similar type of observation that hypertensive patients are more prone to develop epilepsy. Twenty-two of our patients presented with early seizure and 12 presented with late seizure. Early and late seizures are very important to distinguish. Olsen, 2001, found that there is high chance of recurrence rate in late seizure (approximately 50\%), whereas in comparison early seizure has a low recurrence rate (approximately $30 \%$ ).

\section{Conclusion}

Poststroke seizure although not very frequent but important complication after stroke leads to a worse immediate functional outcome and a high long-term mortality rate. Poststroke seizure also leads to secondary neurological injury, including decreased flow of blood in cerebral circulation and increased intracranial pressure. ${ }^{15-17}(29,46,47$ article B). There is lack of adequate data to guide regarding prophylactic usage of AED after stroke. According to American Stroke Association, one should not use prophylactic AED in CVA patients including intra cerebral hemorrhage (ICH). European stroke organization also gives the same recommendations in this regard. However, prophylactic AED may be used depending on the clinical scenario. To conclude, physicians must be very cautious while treating stroke patients regarding development of PSE. Since there are various difficulties in designing and conducting

Impact of DM and HTN on PSE

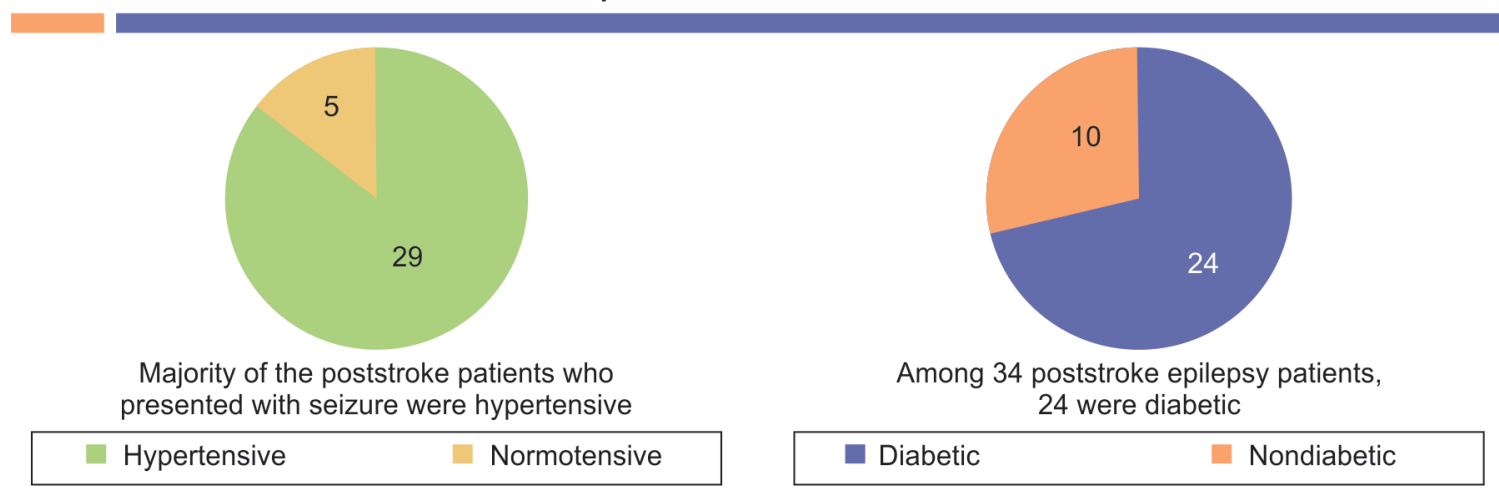

Fig. 7: $85.2 \%$ of our PSE patients were hypertensive 
clinical studies of PSE such as the high diversity of stroke survivors, much more studies are essential to find out primary and secondary preventions of PSE.

\section{References}

1. Zou S, Wu X, Zhu B, et al. The pooled incidence of poststroke seizure in 102008 patients. Top Stroke Rehabil 2015;22(6):460-467. DOI: 10.1 179/1074935715Z.00000000062.

2. Bladin CF, Alexandrov AV, Bellavance A. Seizures after stroke: a prospective multicenter study. Arch Neurol 2000;57(11):1617-1622. DOI: 10.1001/archneur.57.11.1617.

3. Labovitz DL, Hauser WA, Sacco RL. Prevalence and predictors of early seizure and status epilepticus after first stroke. Neurology 2001;57(2):200-206. DOI: 10.1212/WNL.57.2.200.

4. Arboix A, Comes E, Massons J. Relevance of early seizures for in-hospital mortality in acute cerebrovascular disease. Neurology 1996;47(6):1429-1435. DOI: 10.1212/WNL.47.6.1429.

5. Menon B, Shorvon SD. Ischaemic stroke in adults and epilepsy. Epilepsy Res 2009;87(1):1-11. DOI: 10.1016/j.eplepsyres.2009.08.007.

6. Burn J, Dennis M, Bamford J, et al. Epileptic seizures after a first stroke: the Oxfordshire community stroke project. BMJ 1997;315(7122): 1582-1587. DOI: $10.1136 / \mathrm{bmj} .315 .7122 .1582$.

7. Arntz R, Rutten-Jacobs L, Maijwee N, et al. Poststroke epilepsy in young adults: a long term follow up study. PLoS One 2013;8(2):e55498. DOI: 10.1371/journal.pone.0055498.

8. Ferlazzo E, Gasparini S, Beghi E, et al. Epilepsy in cerebrovascular diseases: review of experimental and clinical data with meta-analysis of risk factors. Epilepsia 2016;57(8):1205-1214. DOI: 10.1111/epi.13448.
9. Fisher RS, Acevedo C, Arzimanoglou A, et al. ILAE official report: a practical clinical definition of epilepsy. Epilepsia 2014;55(4):475-482. DOI: 10.1111/epi.12550.

10. Silverman IE, Restrepo L, Mathews GC. Poststroke seizures. Arch Neurol 2002;59(2):195-201. DOI: 10.1001/archneur.59.2.195.

11. Zhang C, Wang X, Wang Y, et al. Risk factors for poststroke seizures: a systematic review and meta analysis. Epilepsy Res 2014;108(10):18061816. DOI: 10.1016/j.eplepsyres.2014.09.030.

12. Graham NSN, Crichton S, Koutroumanidis M, et al. Incidence and associations of poststroke epilepsy the prospective south London stroke register. Stroke 2013;44(3):605-611. DOI: 10.1161/ STROKEAHA.111.000220.

13. Jungehulsing GJ, Heuschmann PU, Holtkamp M, et al. Incidence and predictors of poststroke epilepsy. Acta Neurol Scand 2013;127(6):427430. DOI: 10.1111/ane.12070.

14. Sander JW, Hart YM, Johnson AL, et al. National general practice study of epilepsy:newly diagonosed epileptic seizures in a general population. Lancet 1990;336(8726):1267-1271. DOI: 10.1016/01406736(90)92959-L.

15. Stefanidou M, Das RR, Beiser AS, et al. Incidence of seizures following initial ischaemic stroke in a community based cohort: the Framingham heart study. Seizure 2017;47:105-110. DOI: 10.1016/ j.seizure.2017.03.009.

16. Huang CW, Saposnik G, Fang J, et al. Influence of seizures on stroke outcomes: a large multicenter study. Neurology 2014;82(9):768-776. DOI: 10.1212/WNL.0000000000000166.

17. Goldstein LB, Matchar DB, Morgenlander JC, et al. Influence of drugs on the recovery of sensorimotor function after stroke. J Neurol Rehabil 1990;4:137-144. DOI: 10.1177/136140969000400303. 